This item was submitted to Loughborough's Research Repository by the author.

Items in Figshare are protected by copyright, with all rights reserved, unless otherwise indicated.

\title{
A game-theoretic approach to transmitter covariance matrix design for broadband MIMO Gaussian interference channels
}

PLEASE CITE THE PUBLISHED VERSION

PUBLISHER

(c) IEEE

VERSION

VoR (Version of Record)

\section{LICENCE}

CC BY-NC-ND 4.0

\section{REPOSITORY RECORD}

Anandkumar, Amod J.G., Sangarapillai Lambotharan, and Jonathon Chambers. 2019. "A Game-theoretic Approach to Transmitter Covariance Matrix Design for Broadband MIMO Gaussian Interference Channels". figshare. https://hdl.handle.net/2134/5857. 
This item was submitted to Loughborough's Institutional Repository (https://dspace.lboro.ac.uk/) by the author and is made available under the following Creative Commons Licence conditions.

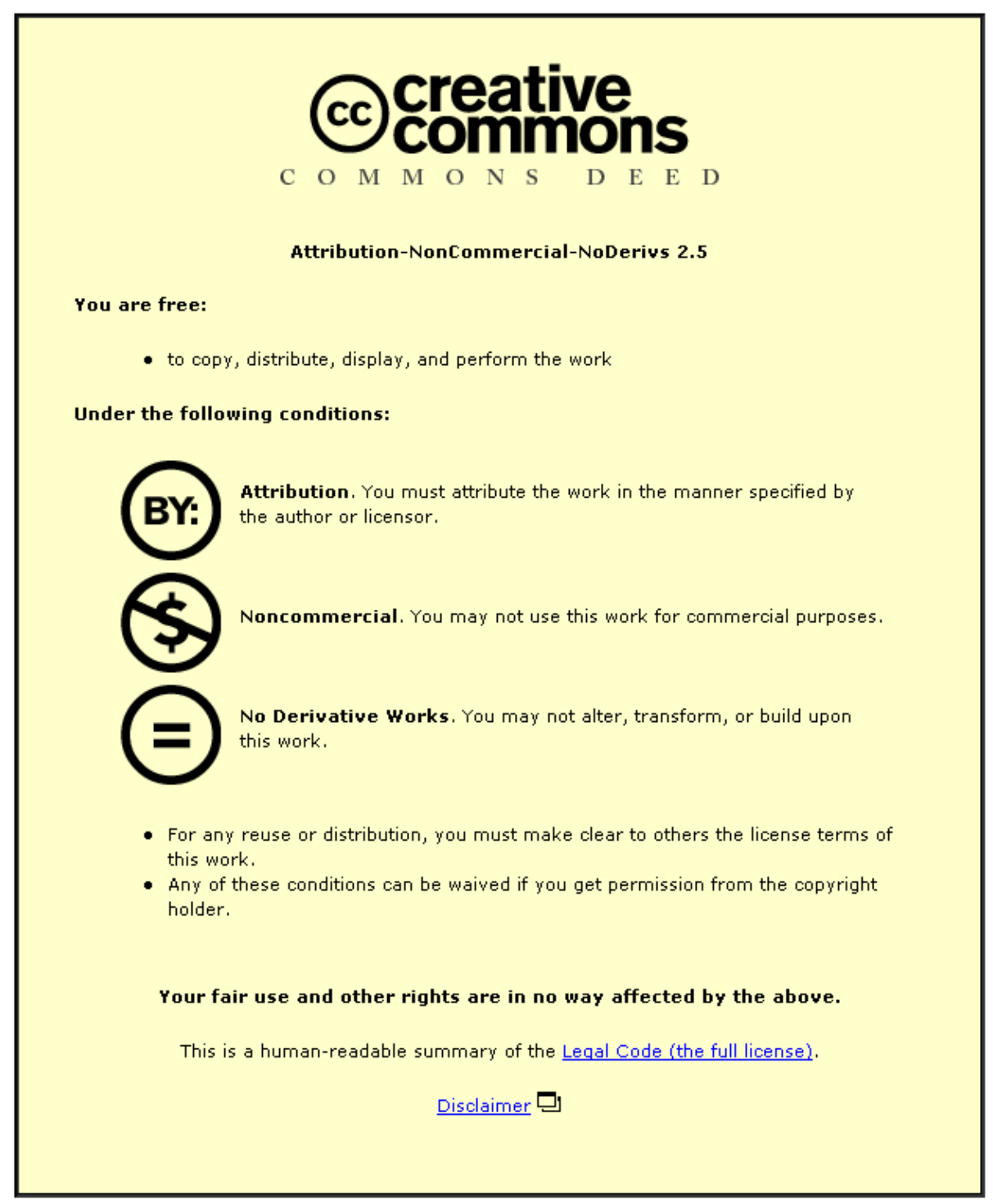

For the full text of this licence, please go to: http://creativecommons.org/licenses/by-nc-nd/2.5/ 


\title{
A GAME-THEORETIC APPROACH TO TRANSMITTER COVARIANCE MATRIX DESIGN FOR BROADBAND MIMO GAUSSIAN INTERFERENCE CHANNELS
}

\author{
A. J. G. Anandkumar, S. Lambotharan, and J. A. Chambers \\ Advanced Signal Processing Group, Dept of Electronic and Electrical Engineering, \\ Loughborough University, Loughborough, U K. LE11 3TU. \\ Email: \{A.J.G.Anandkumar, S. Lambotharan, J.A. Chambers $\}$ @ lboro.ac.uk
}

\begin{abstract}
A game-theoretic approach to the maximization of the information rates of broadband multi-input-multi-output (MIMO) Gaussian interference channels is proposed. The problem is cast as a strategic noncooperative game with the MIMO links as players and the information rates as payoff functions. The Nash equilibrium solution of this game is a waterfilling operation and sufficient conditions for its existence and uniqueness are presented. A distributed algorithm which requires no cooperation among the users is presented along with conditions for guaranteed global convergence of the proposed algorithm. The efficacy of the proposed scheme is confirmed through a design example.
\end{abstract}

Index Terms - Game theory, Nash equilibrium, waterfilling, broadband MU-MIMO systems, Gaussian interference channel

\section{INTRODUCTION}

Game theory allows the modeling of systems where the success of individual decision makers is affected by the actions of other users in the system [1]. This makes it an attractive tool for resource allocation and transmitter power control for many wireless communication systems, for instance, when multiple transmission links in the same environment compete for the use of spectrum to maximize their transmission rates and cause mutual interference. This multi-user (MU) scenario can be modeled as a strategic noncooperative game with selfish players where the links are the players and their information rates are the payoff functions. The advantage of the game-theoretic approach in this scenario is that this multiobjective optimization problem that can only be solved by centralized control is converted into a set of mutually coupled single objective optimization problems. The solution to games of this form is the famous Nash equilibrium [1]. When the players of a game are in Nash equilibrium (NE), no player can increase profits by unilaterally changing strategies. The use of the NE as a competitive optimality criterion can allow this resource allocation problem to be solved with low

This work was supported by EPSRC grant EP/F065477/1 complexity in a distributed fashion without any centralized control. Important issues in the use of the NE as a competitive optimality criterion are the conditions for existence and uniqueness of the NE. Further, fully-distributed algorithms to achieve the NE have to be designed and their convergence conditions have to be characterized.

Related works and contributions: One of the earliest works on multi-user dynamic resource allocation using game theory for vector games was the waterfilling algorithm for digital subscriber line (DSL) systems [2]. An asynchronous fullydistributed waterfilling algorithm based on vector games for SISO Gaussian frequency selective interference channels was presented and characterized in [3]. Detailed studies on the nature of the waterfilling-like Nash equilibrium for SISO Gaussian frequency selective channels were covered in [4] using matrix games and the waterfilling operation was interpreted as the Euclidean projection of a vector onto a convex set in [5] which enabled the derivation of convergence conditions for MU waterfilling algorithms. This work has been extended to narrowband MU-MIMO systems [6], [7] and cognitive radio [8] using matrix game theory. This paper builds on this framework to solve the rate maximization problem for broadband MU-MIMO systems. To the best of our knowledge, the rate maximization problem for broadband MIMO systems has not been solved. The NE for this game, along with its existence and uniqueness conditions are presented. A fully distributed algorithm is proposed along with conditions for guaranteed global convergence. The efficacy of the proposed scheme is confirmed through simulation results using a design example.

The paper is organized as follows: Section 2 describes the system model and the assumptions made in the model. Section 3 is a review of the waterfilling solution for MIMO narrowband channels [6]. Section 4 extends the waterfilling solution to broadband MIMO channels and presents an algorithm to achieve the Nash equilibrium in a distributed manner. Finally, Sections 5 and 6 present the numerical results and conclusions of this work respectively.

Notations used: Vectors and matrices are denoted by lowercase and uppercase boldface letters respectively. The op- 
erators $(\cdot)^{H}, E\{\cdot\}, \operatorname{Tr}(\cdot)$ and $\|\cdot\|_{2}$ are respectively Hermitian, expectation, trace and Euclidean norm operators. The quantity $[\mathbf{A}]_{i j}$ refers to the $(i, j)$ element of $\mathbf{A}$ and $\mathbf{A} \succeq \mathbf{0}$ indicates that $\mathbf{A}$ is positive semidefinite. The sets $\mathbb{C}^{m \times n}$ and $\mathbb{R}_{+}^{m \times n}$ are respectively the set of $m \times n$ matrices with complex and non-negative real elements. The spectral radius of matrix A [9] is denoted by $\rho(\mathbf{A})$ and $(x)^{+} \triangleq \max (0, x)$.

\section{SYSTEM MODEL}

Consider a broadband MIMO Gaussian interference channel with $N$ frequencies, composed of $Q$ MIMO links. At any frequency $f$, the signal vector $\mathbf{y}_{q}^{f} \in \mathbb{C}^{n_{R_{q}} \times 1}$ measured at the receiver of user $q$ is

$$
\mathbf{y}_{q}^{f}=\mathbf{H}_{q q}^{f} \mathbf{x}_{q}^{f}+\sum_{r \neq q} \mathbf{H}_{r q}^{f} \mathbf{x}_{r}^{f}+\mathbf{n}_{q}^{f}
$$

where $\mathbf{H}_{r q}^{f} \in \mathbb{C}^{n_{R_{q}} \times n_{T_{r}}}$ is the channel matrix between source $r$ and destination $q, \mathbf{x}_{q}^{f} \in \mathbb{C}^{n_{T_{q}} \times 1}$ is the vector transmitted by source $q$ and $\mathbf{n}_{q}^{f} \in \mathbb{C}^{n_{R_{q}} \times 1}$ is the receiver noise of user $q$, which is a zero-mean circularly symmetric complex Gaussian vector with an arbitrary (non-singular) covariance matrix $\mathbf{R}_{n_{q}}$. The second term in the right hand side of (1) is the Multi-User Interference (MUI) observed at the destination $q$, which is treated as additive spatially coloured noise. The channel is assumed to be quasi-stationary for the duration of the transmission. At each receiver $q$, the channel matrix $\mathbf{H}_{q q}^{f}$ is assumed to be known and to be square and nonsingular. Also, each receiver is assumed to be able to measure the covariance matrix of the noise plus MUI generated by other users. Based on this information, each destination $q$ computes the optimal covariance matrices $\mathbf{Q}_{q}^{f} \triangleq E\left\{\mathbf{x}^{f} \mathbf{x}^{f^{H}}\right\}$ for each frequency $f$ for its own link and transmits it back to its transmitter in a low bit-rate error-free feedback channel.

\section{MIMO WATERFILLING SOLUTION FOR NARROWBAND CHANNELS}

Consider the system in (1) at a single frequency $f$ as a strategic noncooperative game with the MIMO links as players and information rates of the respective links as payoff functions. Dropping the superscript $f$ for simplicity, the information rate $R_{q}\left(\mathbf{Q}_{q}, \mathbf{Q}_{-q}\right)$ for this system can be written as [6]

$$
R_{q}\left(\mathbf{Q}_{q}, \mathbf{Q}_{-q}\right)=\log \operatorname{det}\left(\mathbf{I}+\mathbf{Q}_{q} \mathbf{H}_{q q}^{H} \mathbf{R}_{-q}^{-1} \mathbf{H}_{q q}\right)
$$

where $\mathbf{R}_{-q} \triangleq \mathbf{R}_{\mathbf{n}_{q}}+\sum_{r \neq q} \mathbf{H}_{r q} \mathbf{Q}_{r} \mathbf{H}_{r q}^{H}$ is the interference plus noise covariance matrix observed by destination $q$, and $\mathbf{Q}_{-q} \triangleq\left\{\mathbf{Q}_{r}\right\}_{r \neq q}^{Q}$ is the set of covariance matrices of all users except the $q$-th user.

Each player $q$ competes rationally against other users in order to maximize its own information rate $R_{q}\left(\mathbf{Q}_{q}, \mathbf{Q}_{-q}\right)$ by designing the optimal covariance matrix $\mathbf{Q}_{q}^{\star}$, given the constraint

$$
E\left\{\left\|\mathbf{x}_{q}\right\|_{2}^{2}\right\} \leq \operatorname{Tr}\left(\mathbf{Q}_{q}\right) \leq P_{q}
$$

where $P_{q}$ is the maximum average power transmitted in units of energy per transmission. Mathematically, the game can be written as

$$
(\mathscr{G} 1):
$$

$$
\begin{array}{ll}
\underset{\mathbf{Q}_{q}}{\operatorname{maximize}} & R_{q}\left(\mathbf{Q}_{q}, \mathbf{Q}_{-q}\right) \\
\text { subject to } & \mathbf{Q}_{q} \in \mathscr{Q}_{q}
\end{array} \forall q \in \Omega
$$

where $\Omega \triangleq\{1, \ldots, Q\}$ is the set of the $Q$ players (i.e. MIMO links), $R_{q}\left(\mathbf{Q}_{q}, \mathbf{Q}_{-q}\right)$ is the payoff function of player $q$ as given in (2) and the set of admissible strategies of player $q$, $\mathscr{Q}_{q}$, is defined as

$$
\mathscr{Q}_{q} \triangleq\left\{\mathbf{Q} \in \mathbb{C}^{n_{T_{q}} \times n_{T_{q}}}: \mathbf{Q} \succeq \mathbf{0}, \quad \operatorname{Tr}\left(\mathbf{Q}_{q}\right)=P_{q}\right\} .
$$

The inequality constraint in (3) is replaced with the equality constraint here as, at the optimum of each problem in (4), the constraint must be satisfied with equality [6]. Further, the players can be limited to pure strategies instead of mixed strategies, as shown in [4]. The solution to the game $\mathscr{G}_{1}$ is the famous Nash equilibrium (NE). At any NE of this game, the optimum action profile of the players $\left\{\mathbf{Q}_{q}^{\star}\right\}_{q \in \Omega}$ must satisfy

$\mathbf{Q}_{q}^{\star}=\mathrm{WF}_{\mathrm{q}}\left(\mathbf{Q}_{1}^{\star}, \ldots, \mathbf{Q}_{q-1}^{\star}, \mathbf{Q}_{q+1}^{\star}, \ldots, \mathbf{Q}_{Q}^{\star}\right)=\mathrm{WF}_{\mathrm{q}}\left(\mathbf{Q}_{-q}^{\star}\right)$.

The waterfilling operator $\mathrm{WF}_{\mathrm{q}}(\cdot)$ is defined as [6]

$$
\mathrm{WF}_{\mathrm{q}}\left(\mathbf{Q}_{-q}\right) \triangleq \mathbf{U}_{q}\left(\mu_{q} \mathbf{I}-\mathbf{D}_{q}^{-1}\right)^{+} \mathbf{U}_{q}^{H}
$$

where $\mu_{q}$ is chosen to satisfy $\operatorname{Tr}\left(\left(\mu_{q} \mathbf{I}-\mathbf{D}_{q}^{-1}\right)^{+}\right)=P_{q}$. The matrices $\mathbf{U}_{q}$ and $\mathbf{D}_{q}$ are calculated from the eigendecomposition

$$
\mathbf{H}_{q q}^{H} \mathbf{R}_{-q}^{-1} \mathbf{H}_{q q} \triangleq \mathbf{U}_{q} \mathbf{D}_{q} \mathbf{U}_{q}^{H}
$$

\section{MIMO WATERFILLING SOLUTION FOR BROADBAND CHANNELS}

\subsection{Problem formulation - the rate maximization game}

In a broadband MIMO system (such as MIMO-OFDM), the power allocation has to be performed across both frequency and space (viz. transmit antennas). This is achieved by designing an optimum covariance matrix for each frequency channel such that the sum of the information rates of all channels is maximized. Defining $\mathcal{Q}_{q}$ as the set of the covariance matrices of player $q$ for the $N$ frequency bins,

$$
\mathcal{Q}_{q} \triangleq\left\{\mathbf{Q}_{q}^{1}, \ldots, \mathbf{Q}_{q}^{N}\right\}, \quad \mathbf{Q}_{q}^{f} \in \mathbb{C}^{n_{T_{q}} \times n_{T_{q}}},
$$

this problem can be cast in the game-theoretic framework as

$$
\begin{array}{lll} 
& \underset{\left.\mathscr{Q}_{2}\right):}{\operatorname{maximize}} & R_{q}\left(\mathcal{Q}_{q}, \mathcal{Q}_{-q}\right) \\
& \text { subject to } & \mathcal{Q}_{q} \in \overline{\mathscr{Q}}_{q}
\end{array} \quad \forall q \in \Omega
$$


where $R_{q}\left(\mathcal{Q}_{q}, \mathcal{Q}_{-q}\right) \triangleq \sum_{f=1}^{N} R_{q}^{f}\left(\mathbf{Q}_{q}^{f}, \mathbf{Q}_{-q}^{f}\right)$ and $R_{q}^{f}\left(\mathbf{Q}_{q}^{f}, \mathbf{Q}_{-q}^{f}\right)$ is defined similar to (2). The set of admissible strategies of player $q, \overline{\mathscr{Q}}_{q}$, is defined as

$$
\overline{\mathscr{Q}}_{q} \triangleq\left\{\mathcal{Q}: \sum_{f=1}^{N} \operatorname{Tr}\left(\mathbf{Q}_{q}^{f}\right)=P_{q} ; \mathbf{Q}_{q}^{f} \succeq \mathbf{0}\right\} .
$$

\subsection{Nash equilibrium of $\mathscr{G}_{2}$}

At any NE of game $\mathscr{G}_{2}$, if it exists, the optimum action profile of the players $\left\{\mathcal{Q}_{q}^{\star}\right\}_{q \in \Omega}$ must satisfy the following simultaneous multi-user waterfilling solutions: $\forall q \in \Omega$,

$$
\mathcal{Q}_{q}^{\star}=\overline{\mathrm{WF}}_{\mathrm{q}}\left(\mathcal{Q}_{1}^{\star}, \ldots, \mathcal{Q}_{q-1}^{\star}, \mathcal{Q}_{q+1}^{\star}, \ldots, \mathcal{Q}_{Q}^{\star}\right)=\overline{\mathrm{WF}}_{\mathrm{q}}\left(\mathcal{Q}_{-q}^{\star}\right) .
$$

The waterfilling operator $\overline{\mathrm{WF}}_{\mathrm{q}}(\cdot)$ is defined as

$$
\overline{\mathrm{WF}}_{\mathrm{q}}\left(\mathcal{Q}_{-q}\right) \triangleq\left\{\mathbf{U}_{q}^{f}\left(\tilde{\mu}_{q} \mathbf{I}-\mathbf{D}_{q}^{f^{-1}}\right)^{+} \mathbf{U}_{q}^{f^{H}}\right\} \quad f=1, \ldots, N
$$

where $\tilde{\mu}_{q}$ is chosen to satisfy $\sum_{f=1}^{N} \operatorname{Tr}\left(\left(\tilde{\mu}_{q} \mathbf{I}-\mathbf{D}_{q}^{f-1}\right)^{+}\right)=$ $P_{q}$. The matrices $\mathbf{U}_{q}^{f}$ and $\mathbf{D}_{q}^{f}$ are calculated from the eigendecomposition

$$
\mathbf{H}_{q q}^{f}{ }^{H} \mathbf{R}_{-q}^{f}{ }^{-1} \mathbf{H}_{q q}^{f} \triangleq \mathbf{U}_{q}^{f} \mathbf{D}_{q}^{f} \mathbf{U}_{q}^{f^{H}} .
$$

\subsection{Existence and uniqueness of Nash equilibrium}

The non-negative matrix $\mathbf{S} \in \mathbb{R}_{+}^{Q \times Q}$ is defined as

$[\mathbf{S}]_{q r} \triangleq\left\{\begin{array}{lr}\max _{f} \rho\left(\mathbf{H}_{r q}^{f}{ }^{H} \mathbf{H}_{q q}^{f}{ }^{H^{-1}} \mathbf{H}_{q q}^{f}{ }^{-1} \mathbf{H}_{r q}^{f}\right), & \text { if } r \neq q, \\ 0, & \text { otherwise. }\end{array}\right.$

The sufficient condition for existence and uniqueness of the $\mathrm{NE}$ of game $\mathscr{G}_{2}$ is given by the following theorem:

Theorem 1 Game $\mathscr{G}_{2}$ has at least one NE for any set of channel matrices and transmit powers of the users. Furthermore, the NE is unique if

$$
\text { (C1): } \quad \rho(\mathbf{S})<1
$$

where $\mathbf{S}$ is defined in (15).

Due to space constraints, the proofs for Theorem 1 (and Theorem 2) have been omitted and will be presented in future publications.

\subsection{Broadband MIMO waterfilling algorithm}

Let $\mathcal{Q}_{q}^{(n)}$ denote the set of covariance matrices of user $q$ at the n-th iteration. A distributed algorithm using sequential update order to reach the NE of game $\mathscr{G}_{2}$ is described in Algorithm 1

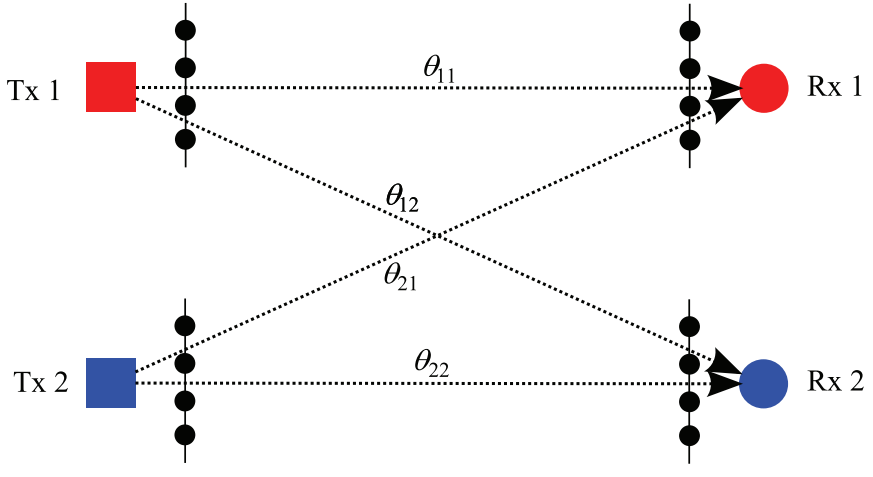

Fig. 1: System with 2 MIMO links having $n_{T}=n_{R}=4$. $\theta_{q r}$ is the electrical angle of the signal observed at destination $r$ from source $q$.

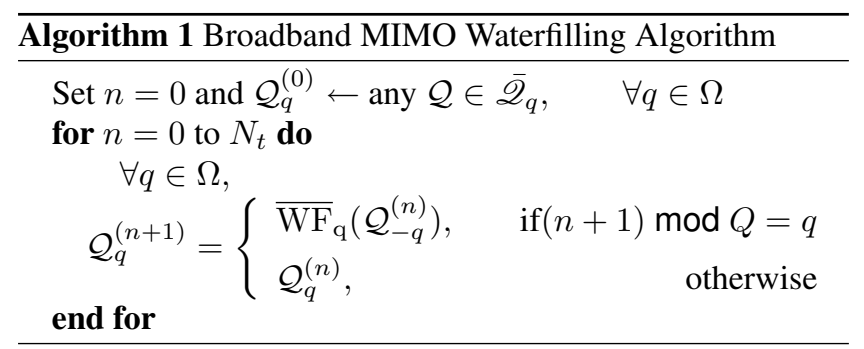

The convergence of Algorithm 1 is guaranteed under the following sufficiency condition:

Theorem 2 The broadband MIMO waterfilling algorithm described in Algorithm 1 converges to the unique NE of game $\mathscr{G}_{2}$ as $N_{t} \rightarrow \infty$ for any set of feasible initial conditions if condition (16) is satisfied.

It is to be noted that Theorem 2 using condition (16) guarantees the global convergence of the broadband MIMO waterfilling algorithm to the unique NE despite game $\mathscr{G}_{2}$ and the waterfilling operation $\overline{\mathrm{WF}}_{\mathrm{q}}(\cdot)$ being nonlinear.

\section{NUMERICAL RESULTS}

To demonstrate the working of the algorithm, a broadband system having 2 MIMO links and 4 frequencies is considered. The source and destination both have 4 antennas placed as a uniform linear array as shown in Figure 1. The signal at destination $r$ from source $q$ is assumed to be at an electrical angle $\theta_{q r}+\epsilon$, where $\epsilon$ is a small Gaussian random variable that varies across different frequencies. This setup allows the demonstration of coherence in the beamformer direction across frequencies. It is to be noted that the algorithm is applicable to any channel, such as MIMO-OFDM channels with multi-path propagation. Figure 2 is the plot of total information rate of each user against iteration index for the system shown in Figure 1, with $\theta_{11}=0, \theta_{12}=-2 * p i / 6, \theta_{21}=p i / 6$ 


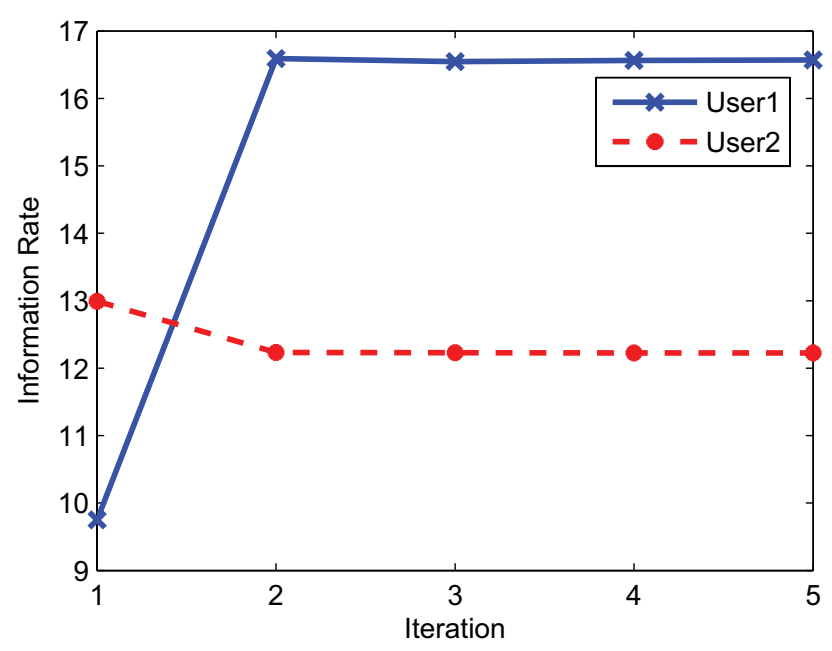

Fig. 2: Information rates of two links with 4 antennas each $\mathrm{v} / \mathrm{s}$ iteration index with $\theta_{11}=0, \theta_{12}=-2 * p i / 6, \theta_{21}=p i / 6$ and $\theta_{22}=2 * p i / 3$.

and $\theta_{22}=2 * p i / 3$. As seen in the figure, the broadband MIMO waterfilling algorithm has very fast convergence. Figure 3 shows the plot of the beamforming patterns associated with the dominant eigenvalues of the optimal covariance matrices computed using the broadband MIMO waterfilling algorithm for the above setup. The major lobes are in the direction of the appropriate receiver at all frequencies for both users. There are no side lobes as the system considered does not have multipath propagation. The fast convergence and appropriate beamformer directions have been observed for many channel realizations and different number of users and frequencies.

\section{CONCLUSIONS}

This paper presented a distributed waterfilling algorithm for resource allocation in broadband MIMO Gaussian interference channels with square (nonsingular) channel matrices. This was done in a game-theoretic framework by considering it as a rate maximization game and the Nash equilibrium for this game was presented. The condition for its existence and uniqueness were presented and a distributed algorithm to reach the Nash equilibrium was demonstrated. The framework proposed in this paper can be extended to broadband MIMO systems with arbitrary channel matrices and other interesting scenarios.

\section{REFERENCES}

[1] M. J. Osborne and A. Rubinstein, A Course in Game Theory, MIT Press, 1999.

[2] W. Yu, G. Ginis, and J.M. Cioffi, "Distributed Multiuser Power Control for Digital Subscriber Lines," IEEE JSAC, vol. 20, no. 5, pp. 1105-1115, Jun 2002.
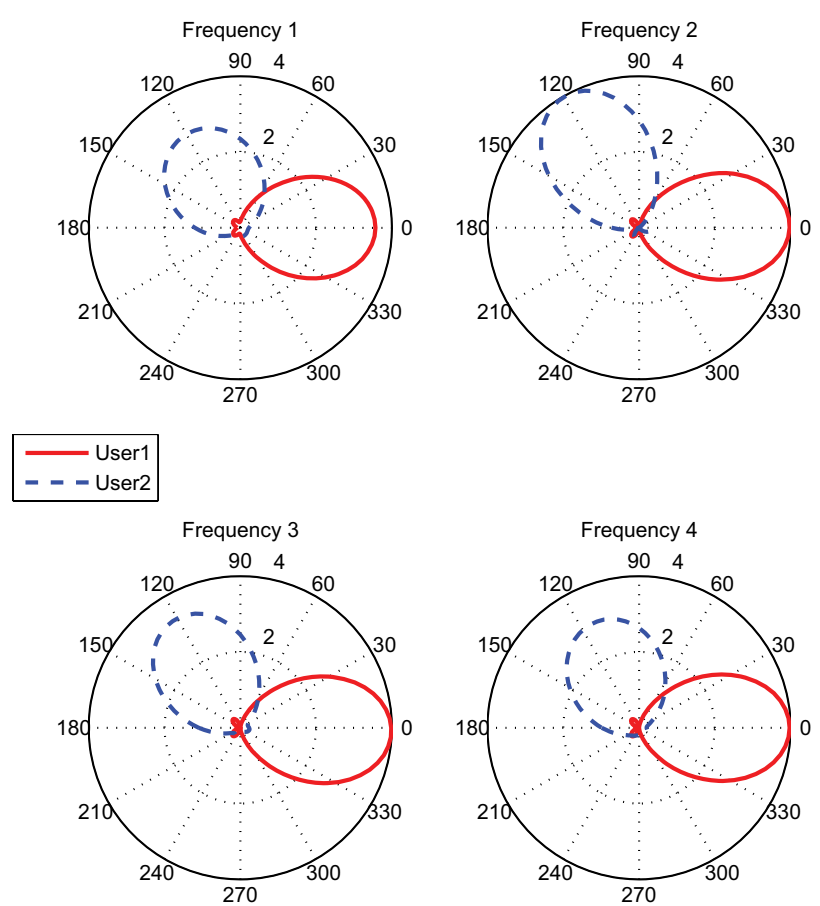

Fig. 3: Beamforming patterns of the two links at the NE of game $\mathscr{G}_{2}$ for the system with $\theta_{11}=p i / 6, \theta_{12}=-2 *$ $p i / 6, \theta_{21}=p i / 6$ and $\theta_{22}=2 * p i / 3$.

[3] G. Scutari, D.P. Palomar, and S. Barbarossa, "Asynchronous Iterative Water-Filling for Gaussian Frequency-Selective Interference Channels," Information Theory, IEEE Trans. on, vol. 54, no. 7, pp. 2868-2878, July 2008.

[4] G. Scutari, D.P. Palomar, and S. Barbarossa, "Optimal Linear Precoding Strategies for Wideband Noncooperative Systems Based on Game Theory-Part I: Nash Equilibria," Signal Processing, IEEE Trans. on, vol. 56, no. 3, pp. 1230-1249, March 2008.

[5] G. Scutari, D.P. Palomar, and S. Barbarossa, "Optimal Linear Precoding Strategies for Wideband Non-Cooperative Systems Based on Game Theory-Part II: Algorithms," Signal Processing, IEEE Trans. on, vol. 56, no. 3, pp. 1250-1267, March 2008.

[6] G. Scutari, D. Palomar, and S. Barbarossa, "Competitive Design of Multiuser MIMO Systems Based on Game Theory: A Unified View," IEEE JSAC, vol. 26, no. 7, pp. 1089-1103, September 2008.

[7] G. Scutari, D.P. Palomar, and S. Barbarossa, "The MIMO Iterative Waterfilling Algorithm," Signal Processing, IEEE Trans. on, accepted for publication.

[8] G. Scutari, D. Palomar, and S. Barbarossa, "Cognitive MIMO Radio," Signal Processing Magazine, IEEE, vol. 25, no. 6, pp. 46-59, November 2008.

[9] R. A. Horn and C. R. Johnson, Matrix Analysis, Cambridge University Press, 1985. 